\title{
Similarity between Hypotheses and Evidence
}

\author{
Yuval Rottenstreich
}

Center for Decision Research, Graduate School of Business, University of Chicago

\author{
Lyle Brenner
}

Warrington College of Business Administration, University of Florida

and

\author{
Sanjay Sood
}

\section{Jones Graduate School of Management, Rice University}

\begin{abstract}
We explore two novel consequences of similarity-based likelihood judgment. In Section I, we distinguish between the evidence on which judgments are based and the hypotheses that serve as the objects of judgment. The location of a feature, whether in the evidence or the hypotheses, influences the perceived similarity between evidence and hypotheses and consequently yields judgments that are inconsistent with the requirements of probability theory. In Section II, we examine judgment of disjunctive hypotheses. For certain types of disjunctions, the assessment of similarity produces consistent nonmonotonicities: the support of a disjunction is smaller than that of one of its components. Finally, we discuss the implications of our findings in terms of support theory and the principle of context independence. (๑) 1999 Academic Press
\end{abstract}

A great deal of research has uncovered numerous effects that arise when assessments of similarity form the basis for judgment under uncertainty. For example, in a classic demonstration of the conjunction fallacy, Tversky and Kahneman (1983) found that an outspoken, socially conscious, and single woman named Linda was judged more likely to be a feminist bank teller than a bank teller, despite the fact that the former category is included within,

This is truly collaborative work, the order of authorship is arbitrary. We thank Maya BarHillel, Derek Koehler, Dale Griffin, Rich Gonzalez, Chip Heath, and Eldar Shafir for helpful discussions. We thank John Pinto and Jennifer Smith for their generous help in conducting the experiments.

Address correspondence and reprint requests to Yuval Rottenstreich, Graduate School of Business, University of Chicago, 1101 East 58th Street, Chicago, IL 60637. Fax: (773) 7020458. E-mail: yuval.rottenstreich@gsb.uchicago.edu. 
and therefore must be less likely than, the latter. The fallacy presumably arises because Linda appears more similar to or better matches a feminist bank teller than a bank teller. A reliance on the assessment of similarity as an input to judgment has also been shown to contribute to base-rate neglect and nonregressive prediction (Tversky \& Kahneman, 1974; Griffin \& Tversky, 1992; Bar-Hillel \& Fischhoff, 1981), insensitivity to sample size (Tversky \& Kahneman, 1971; Borgida \& Nisbett, 1977), illusions of covariation assessment (Jennings, Amabile, \& Ross, 1982; Chapman \& Chapman, 1967, 1969), and misconceptions of random processes (Kahneman \& Tversky, 1972; Gilovich, Vallone, \& Tversky, 1985; Wagenaar \& Bar-Hillel, 1991).

In this paper, we explore two novel consequences of similarity-based likelihood judgment. In Section I we draw on a distinction between the evidence on which judgments are based and the hypotheses which serve as the objects of judgment. In a number of experiments we study how judgment is affected by the location of a particular feature, residing either in the evidence or in the hypotheses. By systematically affecting the degree of similarity or match between evidence and hypotheses, the location of a feature may influence judgment in a manner inconsistent with the requirements of probability theory.

In Section II we examine judgment of disjunctive hypotheses in the presence of specific evidence (case judgments) and in the absence of specific evidence (class judgments). The evaluation of disjunctions may be very different in case judgments, where the perceived similarity between hypotheses and evidence is crucial, and class judgments, where similarity between hypotheses and evidence is not naturally assessed. Thus, contrasting case and class judgments isolates the role of similarity. We predict greater departures from the normative requirement of additivity in similarity-based case judgments than in non-similarity-based class judgments.

In both sections our analysis unfolds in the context of support theory (Tversky \& Koehler, 1994; Rottenstreich \& Tversky, 1997), a recent descriptive model of likelihood judgment. Support theory reduces subjective probability to evaluations of hypothesis strength, which may be based on assessments of similarity. Relating similarity to hypothesis strength allows us to use support theory as a natural framework for incorporating and potentially formally modeling the role of similarity in probability judgment.

It should be recognized that similarity is only one of many mechanisms by which the strength of a hypothesis may be assessed. Similarity is a natural mechanism in judging the likelihood that Linda is a bank teller, for example, but may be far less natural in the judging the likelihood that a cure for AIDS will be discovered before the year 2000. Indeed, much classic work on probability judgment (Tversky \& Kahneman, 1974) emphasizes the notion that different mechanisms are applied to different judgment tasks. Likewise, we should note that the term similarity refers to a collection of psychological mechanisms. For instance, judging the (conceptual) similarity of Linda to a 
bank teller is very different from judging the (perceptual) similarity of an ellipse to a circle. Throughout the present paper our focus is on similarity as manifested in the match or conceptual coherence between hypotheses and evidence. Accordingly, we use the terms similarity and match interchangeably.

\section{LOCATION OF FEATURES}

We first investigate how the location of a feature, whether in the evidence or in the hypotheses, may influence judgment. Consider the following slightly updated version of Kahneman and Tversky's character Linda:

Problem 1E. Linda is 31 years old, single, outspoken, and very bright. In college, she majored in philosophy. As a student she was deeply concerned with issues of discrimination and social justice, and also participated in anti-apartheid demonstrations. Linda is active in the feminist movement.

One of the two statements below is also true of Linda. Please estimate the probability of each, making sure that the probabilities sum to $100 \%$.

(a) Linda is a journalist.

(b) Linda is an insurance salesperson.

We can represent the conditional judgments of whether Linda is a journalist or an insurance salesperson as $P\left(H_{i} \mid E\right)$. In this notation, $H_{i}$ refers to the hypothesis being judged, with journalist as $H_{1}$ and insurance salesperson as $H_{2}$. $E$ refers to the set of evidence on which the evaluation of each hypothesis depends - in this case the collection of features describing Linda.

Probability theory makes no fundamental distinction between hypotheses and evidence; both are simply defined as subsets of the relevant sample space. Any subset may serve equally well either as a conditioning event (i.e., as evidence) or as an event evaluated conditional on another (i.e., as a hypothesis). Thus, whether a given feature is located in the evidence or in the hypotheses is irrelevant, in the following formal sense. Let $E_{0}$ and $F$ denote two sets of features. A simple application of the definition of conditional probability yields the following equality:

$$
\frac{P\left(H_{1} \mid E_{0} \wedge F\right)}{P\left(H_{2} \mid E_{0} \wedge F\right)}=\frac{P\left(H_{1} \wedge F \mid E_{0}\right)}{P\left(H_{2} \wedge F \mid E_{0}\right)} .
$$

That is, the relative likelihood of $H_{1}$ and $H_{2}$ given $E_{0}$ should not depend on whether $F$ resides in the evidence or is paired with $H_{1}$ and $H_{2}$ in the hypotheses. ${ }^{1}$

\footnotetext{
${ }^{1}$ Note that Eq. (1) applies to ratios of probabilities, not to the corresponding numerators and denominators separately. In cases such as Problem 1E, where there are only two hypotheses under consideration, this distinction is irrelevant. With only two hypotheses under consideration, specifying either the numerator or the denominator also specifies the other (the two
} 
Contrary to Eq. (1), however, if judged probabilities are based primarily on an assessment of similarity, the location of a particular feature may be critically important. Consider changing Problem 1E by moving the feature Linda is active in the feminist movement from the evidence to the hypotheses to form Problem 1H:

Problem $1 H$. Linda is 31 years old, single, outspoken, and very bright. She majored in philosophy. As a student she was deeply concerned with issues of discrimination and social justice, and also participated in anti-apartheid demonstrations.

One of the two statements below is also true of Linda. Please estimate the probability of each, making sure that the probabilities sum to $100 \%$.

(a) Linda is active in the feminist movement and is a journalist.

(b) Linda is active in the feminist movement and is an insurance salesperson.

Consider how the similarity between evidence and hypotheses is affected as feminist is moved from one to the other. In Problem 1E, the hypothesis journalist is highly similar to the evidence feminist Linda, and in Problem $1 \mathrm{H}$, the hypothesis feminist journalist remains highly similar to the evidence Linda. Because feminist is largely consistent both with Linda and with journalist, in this case moving the feature feminist has little effect on the overall degree of match between hypotheses and evidence. On the other hand, in Problem 1E, the hypothesis insurance salesperson appears not at all similar to feminist Linda, but in Problem $1 \mathrm{H}$ the hypothesis feminist insurance salesperson is somewhat more similar to Linda. Because feminist is consistent with Linda but not with journalist, in this case moving the feature feminist has a substantial effect on the overall degree of match between hypotheses and evidence.

The asymmetric changes in similarity that result from moving the target feature are predicted to be reflected in probability judgments. Contrary to the requirement specified by Eq. (1), as feminist is moved from the evidence to the hypotheses, the judged likelihood of insurance salesperson may increase relative to the judged likelihood of journalist.

Support theory allows for a simple characterization of this possibility. In support theory, each hypothesis $A$ has a support value $s(A)$, corresponding to the strength of evidence for that hypothesis. The judged probability, $P(A$, $B$ ), that $A$ holds rather than $B$ assuming one and only one obtains, is given by $P(A, B)=s(A) /[s(A)+s(B)]$. The theory thus reduces probability judgment to the assessment of support. In the tasks we study, we assume that support is driven largely by assessments of the similarity or match between hypotheses and evidence. 
Using support theory we can represent the judgment when $F$ is in the evidence as

$$
P\left(H_{1}, H_{2} \mid E_{0} \wedge F\right)=\frac{s_{\text {ef }}\left(H_{1}\right)}{s_{\text {ef }}\left(H_{1}\right)+s_{\text {ef }}\left(H_{2}\right)} .
$$

Here, the scale $s_{\text {ef }}$ represents the degree of support based on the set of evidence $E_{0} \wedge F$. We can represent the judgment when the common feature is in both hypotheses as

$$
P\left(H_{1} \wedge F, H_{2} \wedge F \mid E_{0}\right)=\frac{s_{\mathrm{e}}\left(H_{1} \wedge F\right)}{s_{\mathrm{e}}\left(H_{1} \wedge F\right)+s_{\mathrm{e}}\left(H_{2} \wedge F\right)}
$$

Here $s_{\mathrm{e}}$ represents the degree of support based on the evidence $E_{0}$. With these formulations it is easy to see that Eq. (1) will be satisfied whenever $s_{\text {ef }}\left(H_{1}\right) /$ $s_{\mathrm{e}}\left(H_{1} \wedge F\right)=s_{\mathrm{ef}}\left(H_{2}\right) / s_{\mathrm{e}}\left(H_{2} \wedge F\right)$ - that is, when the proportional change in support for $H_{1}$ and $H_{2}$ is constant as the critical feature is moved from the evidence to the hypotheses.

As suggested by Problem 1, however, if support is based largely on similarity, this condition is unlikely to hold. Specifically, moving a particular feature from the evidence to the hypotheses may asymmetrically affect the support for (initially) weak and strong hypotheses. When, as in the Linda example, the moved feature $F$ matches the remaining evidence $E_{0}$, we should find that as both hypotheses are paired with $F$, the weaker hypothesis (insurance salesperson) is strengthened relative to the stronger hypothesis (journalist). We test this prediction in the following study.

\section{Study 1: Relocation of Features Matching the Evidence}

Students at Stanford University $(n=46)$ completed a short questionnaire containing two problems, one involving Linda (either Problem 1E or Problem $1 \mathrm{H}$ ) and a second involving a version of Tversky and Kahneman's wellknown character Bill:

Problem 2. Bill is 34 years old. He is intelligent, but unimaginative, compulsive, and generally lifeless. In school he was strong in mathematics but weak in social studies and humanities. [Bill is an accountant.]

One of the two statements below is also true of Bill. Please estimate the probability of each, making sure that the probabilities sum to $100 \%$.

(a) Bill [is an accountant and] plays bridge as a hobby.

(b) Bill [is an accountant and] plays jazz as a hobby.

Problem 2E included the bracketed text in the description of Bill, while Problem $2 \mathrm{H}$ included the bracketed text in the hypotheses. Subjects encountered either Problems $1 \mathrm{E}$ and $2 \mathrm{H}$ or Problems $1 \mathrm{H}$ and $2 \mathrm{E}$. Within each version of each problem, the order of the hypotheses was counterbalanced. 
We predicted that in Problems 1 and 2, pairing the weak hypotheses (insurance salesperson and plays jazz, respectively) with a feature consistent with the remaining evidence (feminist and accountant, respectively) would increase the judged probabilities of these hypotheses. In accordance with this prediction, the median judged probability of the weak hypotheses was greater when the feature resided in the hypotheses (the $\mathrm{H}$ versions) than in the evidence (the E versions) for both problems, 37 vs. $27 \%$ for Linda and 40 vs. $30 \%$ for Bill (Mann-Whitney $p<.05$ ).

We also studied problems where the stronger, matching hypothesis was defined in terms of the weaker, nonmatching hypothesis. Seventy-six California Institute of Technology students read revisions of the two Linda problems in which insurance salesperson was paired with not an insurance salesperson (instead of journalist) and revisions of the two Bill problems in which plays jazz was paired with does not play jazz (instead of plays bridge). Defining the stronger hypothesis in terms of the weaker hypothesis is of interest for two reasons. First, such definitions eliminate the possibly confusing requirement that the judge condition on two occupations or hobbies to the exclusion of all others. Second, such definitions essentially entail an assessment of the similarity of just one object, either insurance salesperson or plays jazz, to the relevant evidence.

Despite these differences, the prediction based on asymmetric changes in the similarity between hypotheses and evidence remains the same; support for the weaker hypothesis should be relatively greater when the critical feature is located in the hypotheses. Indeed, judgments for the revised problems yielded the same pattern of results observed for the original problems. The median judged probability of the weaker hypotheses was greater in the $\mathrm{H}$ versions than in the E versions for both problems, 35 vs. $25 \%$ for Linda and 30 vs. $18 \%$ for Bill (Mann-Whitney $p<.05$ ).

\section{Study 2: Relocation of Features Not Matching the Evidence}

In the previous demonstrations, pairing an evidence-matching feature with both a weaker and a stronger hypothesis increased the judged probability of the weaker hypothesis. The reverse effect, a decrease in the judged probability of the weaker hypothesis, would be expected when both hypotheses are paired with a feature that does not match the evidence. For example

Problem 3. Linda is 31 years old, single, outspoken, and very bright. In college, she majored in philosophy. As a student she was deeply concerned with issues of discrimination and social justice, and also participated in anti-apartheid demonstrations. [After college Linda studied cinematography.]

One of the two statements below is also true of Linda. Please estimate the probability of each, making sure the probabilities sum to $100 \%$.

(a) [After college Linda studied cinematography, and she] Linda is now a documentary filmmaker.

(b) [After college Linda studied cinematography, and she] Linda now directs music videos. 
Problem 3E included the bracketed text in the description of Linda, while Problem $3 \mathrm{H}$ included the bracketed text in the hypotheses. Consider how moving the feature studied cinematography changes the similarity between the description of Linda and the hypotheses. In this case Linda who studied cinematography is similar to documentary filmmaker, and Linda is also similar to studied cinematography and documentary filmmaker. On the other hand, Linda who studied cinematography is somewhat similar to directs music videos; but Linda is not at all similar to studied cinematography and directs music videos. The decrease in similarity occurs because studied cinematography is the primary match for directs music videos, and moving that feature to the hypotheses results in essentially no match between the resulting conjunction and the remaining evidence.

The asymmetric changes in similarity should be reflected in probability judgment. As studied cinematography is moved from the evidence to the hypotheses, the judged likelihood of directs music videos should decrease relative to the judged likelihood of documentary filmmaker. Thus, in this case, where the moved feature does not match the remaining evidence, we expect precisely the opposite of the pattern observed when the moved feature matched the remaining evidence.

To test this prediction we presented 47 students at Stanford University with a questionnaire containing two problems. The first was either Problem $3 \mathrm{E}$ or Problem $3 \mathrm{H}$ involving Linda. The second was another problem involving Bill:

Problem 4. Bill is 34 years old. He is intelligent, but unimaginative, compulsive, and generally lifeless. In school he was strong in mathematics but weak in social studies and humanities. [Every Thursday Bill gets together with three of his friends.]

One of the two statements below is also true of Bill. Please estimate the probability of each, making sure that the probabilities sum to $100 \%$.

(a) [Every Thursday Bill gets together with three of his friends.] Bill and his friends play bridge as a hobby.

(b) [Every Thursday Bill gets together with three of his friends.] Bill and his friends play jazz as a hobby.

As before, subjects encountered one feature in evidence (E version) problem and one feature in hypotheses (H version) problem. Within each version of each problem, the order of the hypotheses was counterbalanced.

The judged probabilities are again consistent with the predictions based on considerations of similarity. The median judged probabilities of the weaker hypotheses were greater in the $\mathrm{E}$ versions than in the $\mathrm{H}$ versions for both problems, 30 vs. $20 \%$ for Linda and 27 vs. 20\% for Bill (Mann-Whitney $p<.05)$.

\section{Discussion}

Our findings show that movement of a feature from evidence to two competing hypotheses does not have a proportionally equivalent effect on the 
support for both hypotheses, as required by Eq. (1). Instead, the moved feature asymmetrically affects weak and strong hypotheses, consistent with the notion that judgment is based on similarity between evidence and hypotheses.

A key aspect of the situations we have studied is that similarity of the stronger hypothesis $H_{1}$ to the evidence does not change very much, because of an abundance of matching features between $H_{1}$ and $E_{0}$. Locating a partially redundant feature $F$ in either the hypotheses or the evidence has little effect on $H_{1}$ because the match between it and the evidence is already high. The similarity of the weaker hypothesis $H_{2}$ to the evidence, in contrast, is highly dependent on the location of $F$, precisely because $H_{2}$ does not match $E_{0}$ very well. In the first set of demonstrations, $\mathrm{H}_{2}$ (e.g., insurance salesperson) receives greater support when a feature that matches $E_{0}$ (e.g., feminist) is also included in the hypothesis. In the second set of demonstrations, $H_{2}$ (e.g., directs music videos) receives greater support when a feature that matches $\mathrm{H}_{2}$, but does not match the rest of the evidence (e.g., studied cinematography), is located in the evidence.

These results rule out two possible psychological processes. First, one could 'transfer'" features common to all hypotheses out of the hypotheses and consider these common features as if they were part of the evidence. Second, although it leads to violations of probability theory, one could simply ignore or "cancel" features common to all hypotheses. In principle, both transfer and cancellation appear to be attractive procedures; each reduces the complexity of the judgment task. In Problem $1 \mathrm{H}$, for instance, both transfer and cancellation would reduce the evaluation of compound hypotheses (e.g., feminist journalist) to the evaluation of simple hypotheses (e.g., journalist).

Nevertheless, neither transfer nor cancellation appears to characterize participants' responses to Problems 1 through 4. Transfer entails judgments that satisfy Eq. (1) and is thus ruled out by the differences between observed judgments in the $\mathrm{E}$ and $\mathrm{H}$ versions of all four problems. These differences also cast doubt on the validity of cancellation. It seems plausible that judgments of journalist versus insurance salesperson based on a description of Linda including the feature feminist (as in the E version) would be roughly equivalent to judgments based on a description of Linda not including the feature feminist (as in the $\mathrm{H}$ version after cancellation). This conjecture is based on the notion that feminist is largely redundant with the other features of Linda. Had subjects canceled the common feature, however, we would have observed little or no difference between the $\mathrm{E}$ and $\mathrm{H}$ versions of the problems.

Rather than identifying features common across hypotheses and then either transferring or canceling, respondents appear to judge the similarity between each hypothesis and the evidence individually. In effect, the support for each hypothesis is considered independently, without reference to the 
other hypothesis. That is, this pattern reveals across-hypothesis context independence.

We should also note that the results of Problems 1 through 4 rule out simple averaging models in which the support or probability of a conjunction is the average of the supports or probabilities assigned to its components. Such models would predict that in all four problems judged probabilities should regress toward a 50/50 split as a particular feature is moved out of the evidence and placed in both hypotheses. While this pattern was observed in the first and second problems, precisely the opposite pattern was observed in the third and fourth problems.

There are many situations in which the location of a particular feature may have important consequences. Consider for example a murder trial in which guilt and innocence of the defendant are the possible hypotheses. Moreover, suppose that much of the evidence implicates the defendant: the defendant's ex-wife is the victim, the two were seen arguing hours before the murder took place, and the defendant's alibi cannot be substantiated. Furthermore, traces of the victim's blood were found in the defendant's sport-utility vehicle and in the defendant's driveway. On the other hand, a glove possibly used by the murderer does not fit the defendant. Under these circumstances, we suggest that many people would see the weight of the evidence as indicating an extremely high likelihood of the defendant's guilt. The great majority of the evidence-all but one piece-matches guilt much better than innocence.

Suppose, however, that the lone divergent fact is moved from the evidence to both hypotheses, so that one now judges the hypothesis the glove doesn't fit and the defendant is guilty against the hypothesis the glove doesn't fit and the defendant is innocent. In this case, every one of the remaining facts in evidence is at odds with a critical feature present in both hypotheses. Each hypothesis may thus seem less likely than before. More importantly, however, we suggest that many people may now view the relative likelihood of the hypothesis involving guilt as substantially more moderate. The evidence no longer forms an overwhelmingly better match to the hypothesis involving guilt compared to the hypothesis involving innocence.

The previous analysis suggests that the interested parties in such a legal case would have competing incentives to present this problem with the critical glove feature either in the hypotheses or in the evidence. The defendant's attorneys would want to frame the situation so that jurors consider the glove feature as part of the hypotheses. At the same time, prosecutors would want to encourage jurors to see the glove fact as just another piece of evidence. The outcome of a case could very possibly depend on which side is more successful in locating the critical feature in the evidence or the hypotheses. 


\section{CLASS AND CASE JUDGMENTS OF DISJUNCTIONS}

In Section I we noted that changing the location of a feature may asymmetrically affect the similarity between hypotheses and evidence and yield a pattern of likelihood judgments inconsistent with probability theory. In the present section we examine how similarity is affected when singleton hypotheses are combined to form disjunctions. We observe that when singletons are combined, the similarity between hypotheses and evidence is affected in a manner which also yields judgments inconsistent with the predictions of probability theory.

To isolate the role of similarity in the judgment of disjunctions, we contrast case judgments with class judgments. In case judgments, specific evidence (e.g., the description of Linda) is provided and an evaluation of the match between hypotheses (e.g., that Linda is a journalist) and evidence naturally arises. In class judgments, on the other hand, no specific evidence is provided. For example, a judge might be asked to assess the probability that a randomly selected American is a journalist. Therefore, similarity between evidence and hypotheses cannot be evaluated, and mechanisms other than similarity presumably underlie judgment.

We are concerned with how the similarity between the evidence and each component of the disjunction relates to the similarity between the evidence and the disjunction as a whole. Suppose that, given a description of Linda, a judge is asked to estimate the probability of the disjunctive hypothesis that Linda works as either a journalist or a realtor. We suggest that Linda may appear highly similar to journalist, but less similar to the disjunction journalist or realtor (see also Bar-Hillel \& Neter, 1993). If support is based on similarity, this analysis implies that in case judgments support for disjunctions may be nonmonotonic. In terms of our example, s(journalist or realtor $)<s$ (journalist $)$.

A different pattern may arise in class judgment. Suppose a judge is asked to estimate the relative frequency of the disjunction journalists or realtors as occupations in the United States. The judge may make this judgment by initially assessing, say, the relative frequency of journalists and then adjusting upward to account for additional people who work as realtors (see Rottenstreich \& Tversky, 1997). Because adjustments of this sort are typically insufficient (Slovic \& Lichtenstein, 1968; Tversky \& Kahneman, 1974), this analysis implies that in class judgments support for disjunctions may be monotonic but subadditive. For example, $s$ (journalist or realtor) $>$ $s($ journalist $)$ but $s($ journalist or realtor $)<s($ journalist $)+s($ realtor $)$. Contrasting case judgments with class judgments thus allows us to determine whether assessments of similarity are central to nonmonotonicity of support.

Our predictions for case and class disjunctions can be tested using estimates of support derived from probability judgments. Probability theory re- 
quires additivity of support for disjunctions: for any disjoint hypotheses $H_{1}$ and $H_{2}, s\left(H_{1} \vee H_{2}\right)=s\left(H_{1}\right)+s\left(H_{2}\right)$. However, for class judgments we predict $s\left(H_{1} \vee H_{2}\right) \leq s\left(H_{1}\right)+s\left(H_{2}\right)$ and for case judgment we predict the even more extreme pattern $s\left(H_{1} \vee H_{2}\right) \leq s\left(H_{1}\right)$.

\section{Study 3: Case and Class Disjunctions}

Participants were 177 UCLA undergraduates who completed a short questionnaire within a larger packet containing several unrelated tasks. Approximately half the participants made case judgments and half made class judgments.

Participants made judgments for two problems. In the first problem, participants making class judgments were asked to consider all California residents who work in one of three occupations: journalist $(A)$, insurance salesperson $\left(B_{1}\right)$, or realtor $\left(B_{2}\right)$. One group of participants estimated the relative frequencies of each of the three occupations, providing $P(A), P\left(B_{1}\right)$, and $P\left(B_{2}\right)$, such that the total was $100 \%$. Another group of participants split the total $100 \%$ across the singleton $A$ and the disjunction $B_{1} \vee B_{2}$. These judgments are denoted $P\left(A, B_{1} \vee B_{2}\right)$ and $P\left(B_{1} \vee B_{2}, A\right)$, respectively. A third group judged $P\left(A \vee B_{1}, B_{2}\right)$ and $P\left(B_{2}, A \vee B_{1}\right)$, and a fourth group judged $P\left(A \vee B_{2}, B_{1}\right)$ and $P\left(B_{1}, A \vee B_{2}\right)$. The second problem was similar in structure to the first and concerned accountants $(A)$, bartenders $\left(B_{1}\right)$, and waiters $\left(B_{2}\right)$.

Participants making case judgments judged the likelihood that a target character held one occupation (or disjunction of occupations) rather than another. The first problem included a description of Linda who was said to work as either a journalist $(A)$, insurance salesperson $\left(B_{1}\right)$, or realtor $\left(B_{2}\right)$. As in the class judgments, participants partitioned $100 \%$ probability either across the three hypotheses $A, B_{1}$, and $B_{2}$; across the two hypotheses $A$ and $B_{1} \vee B_{2}$, across $B_{1}$ and $A \vee B_{2}$, or across $B_{2}$ and $A \vee B_{1}$. The second problem included a description of Bill who was said to work as either an accountant, bartender, or waiter.

Estimation of support. Because support is a ratio scale, for each problem in either the case or class format we can set $s(A)=1$ without loss of generality. We can then solve for the remaining support values. For instance, we can solve for the support value of either $B_{1}$ or $B_{2}$ in the condition involving a three-way split of probability by noting that according to support theory:

$$
P\left(B_{\mathrm{i}}\right) / P(A)=s\left(B_{i}\right) / s(A) .
$$

Because the two terms on the left-hand side refer to observed data and, by definition, $s(A)=1, s\left(B_{i}\right)$ is uniquely determined. The support of a disjunction, for example, $A \vee B_{2}$, can then be obtained from the equation

$$
P\left(A \vee B_{2}, B_{1}\right) / P\left(B_{1}, A \vee B_{2}\right)=s\left(A \vee B_{2}\right) / s\left(B_{1}\right)
$$


To take a concrete example, for the Linda problem in the case format, the mean judged probabilities for journalist, insurance salesperson, and realtor in the triple condition were 65,16 , and $19 \%$, respectively. Setting the support for journalist equal to 1, the support for insurance salesperson is then .25 (i.e., 16/65) and the support of realtor is .29 (i.e., 19/65). Furthermore, when the singleton insurance salesperson was paired with the disjunction journalist or realtor, the mean judged probabilities of these two hypotheses were 29 and $71 \%$ respectively. The support for journalist or realtor is thus .62 (i.e., $(71 / 29) \times .25)$.

The other support values in Table 1 are calculated similarly, using the mean judged probabilities for each version of each problem. A simple summary of the results is provided by examining subadditivity weights, denoted by $w$. The subadditivity weight is the ratio of the support for a disjunction compared to the sum of the supports of its individual components. For example, for the Linda problem in the case format, $w$ of the disjunction journalist or realtor is $.62 /(1.00+.29)=.48$. Under additivity, $w$ should be equal to 1 . However, if support for disjunctions is subadditive, $w$ will be less than 1 .

Consistent with the general prediction of subadditivity, the $w$ 's derived from both case and class formats are all less than 1 (see Rottenstreich \& Tversky, 1997, for earlier results on subadditivity for class disjunctions). Moreover, they are generally smaller for case judgments (average $w=.61$ ) than for class judgments (average $w=.82, z=2.03, p<.05){ }^{2}$ This pattern holds for all six individual case-class comparisons. ${ }^{3}$

The results also indicate that in case judgments the support of a disjunction often violates monotonicity. For example, the support of the case disjunction journalist or realtor (.62) is less than the support of journalist (1.00). Four of the six case disjunctions violate monotonicity, while all six class disjunctions satisfy monotonicity.

Interestingly, the four case disjunctions that violate monotonicity all involve one strong, matching hypothesis (e.g., journalist resembles Linda) and one weak, nonmatching hypothesis (e.g., realtor does not resemble Linda). Case judgments of disjunctions involving two weak hypotheses (e.g., realtor and insurance salesperson) yield subadditivity but monotonicity of support.

The observation of a divergence between strong-weak and weak-weak

${ }^{2}$ The standard errors for $w$ 's based on the mean judgments are difficult to determine because the calculation involves division. Thus, individual judgments were converted to logs, and $\log (w)$ was calculated for each disjunction by subtracting the appropriate differences in the mean $\operatorname{logs}$. The standard errors for the resulting $\log (w)$ 's are then easily calculated, and the test involves a straightforward normal approximation.

${ }^{3}$ As another measure of subadditivity, note that under additivity the sum of the judgments across the three disjunctions should be $200 \%$ (because each of the singletons appears in two disjunctions). However, the average sum is $173 \%$ for case judgments and $186 \%$ for class judgments, indicating that disjunctions are receiving less support than would be expected under additivity (for similar results see also Rottenstreich \& Tversky, 1997). 





case disjunctions reveals one advantage of a support-based analysis. Note that nonmonotonicity of support does not imply nonmonotonicity of probabilities. For example, in case judgments the support of journalist or insurance salesperson is smaller than the support of journalist, despite the fact that the judged probability of journalist or insurance salesperson is slightly greater than that of journalist. Nonmonotonicities of support produce nonmonotonicities of probability only when they are sufficiently extreme. Thus, whereas an analysis in terms of support has drawn a distinction between judgments of strong-weak and weak-weak disjunctions, an analysis based directly on probability judgments would not have done so. The distinction between different types of disjunctions may be important, because the judgment of each type of disjunction may have unique psychological properties. Accordingly, in the following study we extend the present results by comparing strong-weak and strong-strong disjunctions.

\section{Study 4: Two Strong Hypotheses and a Weak Hypothesis}

Participants were 216 University of Chicago students, who completed a short questionnaire in exchange for $\$ 1$. They were given problems similar to those in the previous study, with two changes. First, participants making class judgments judged the relative frequency of occupations in Illinois rather than California. Second, the occupations under consideration were changed. In the Linda problem, the three occupations were social worker, public defender, and realtor. In the Bill problem, the three occupations were computer programmer, statistician, and waiter. In each case, two of the occupations now provide a relatively strong match to the target character, and the remaining occupation provides a relatively weak match.

The results, summarized in Table 2, reveal substantial subadditivity. The w's in both formats again tend to be less than 1 . Once more, they are generally smaller for case judgments (average $w=.53$ ) than for class judgments (average $w=.95, z=4.85, p<.001) .{ }^{4}$ Furthermore, in case judgments five of the six disjunctions violate monotonicity, while in class judgments only one of the six disjunctions violates monotonicity.

Our results regarding monotonicity can be summarized as follows. Across Studies 3 and 4 we find monotonicity for weak-weak case disjunctions (Study 3), nonmonotonicity for strong-strong case disjunctions (Study 4), and nonmonotonicity for strong-weak case disjunctions (Studies 3 and 4). On the other hand, across the two studies we consistently find monotonicity for all three types of class disjunctions.

\section{Discussion}

The subadditivity observed for all types of case disjunctions indicates that there is a discrepancy between the similarity of a disjunction to the evidence 





and the similarity of the individual components to the evidence. This discrepancy may be the result of several processes. First, as noted above, strong similarity of one component (e.g., journalist) may be diluted by the addition of nonmatching components (e.g., realtor). This process can explain nonmonotonicity of support for disjunctions involving one strong and one weak component.

Second, components of the disjunction may modify or interact with each other and lead to potentially different impressions of each component. For example, the disjunction public defender or social worker may perhaps call to mind particularly argumentative social workers or particularly idealistic public defenders. Overall support for the disjunction may decrease to the extent that modifications of the individual components limit the scope of the disjunction or reduce the match between the hypothesis and the evidence. Nonmonotonicity of support for strong-strong disjunctions may result from such interactions between components of disjunctions. The extreme degree of nonmonotonicity for case judgments of strong-strong disjunctions (in both problems, the support of the strong-strong disjunction is smaller than the support assigned to either of its individual components) is consistent with this account.

The relatively mild degree of subadditivity (and monotonicity of support) observed in case judgments of weak-weak disjunctions is also worthy of note. We suggest that in disjunctions involving two weak components, people may be performing operations similar to those in class judgments. For example, because of the complete mismatch between the evidence and the components of the disjunction, one may note that Linda looks nothing like a realtor, but that there are many realtors in the population (and analogously for insurance salespersons). One component of the disjunction may serve as an anchor and upward adjustments may be made for the second component, resulting in monotonicity but subadditivity. Put differently, when evaluating weak hypotheses, there is little similarity to be assessed, and case judgment may resemble class judgment. The relatively small and nearly equal degree of subadditivity for case and class judgments of weak-weak disjunctions in Study 3 is consistent with this account (see Table 1).

In summary, different processes may be at work in the judgment of different types of disjunctions. Pairing two weak hypotheses together resulted in subadditivity but not violations of monotonicity. Since by definition the degree of match with weak hypotheses is low, similarity may not play much of a role so that these judgments parallel those of class judgments (i.e., subadditive but monotonic). Second, pairing a weak hypothesis with a strong hypothesis resulted in both subadditivity and nonmonotonicity. Evidently, adding a weak hypothesis dilutes the degree of match or support for the strong hypothesis. Finally, pairing two strong hypotheses together also resulted in both subadditivity and nonmonotonicity. This result may emerge because 
strong hypotheses modify each other in a way that produces a very different and relatively ill-matching composite image.

\section{General Discussion and Conclusions}

In Section I, we observed that the location of a particular feature can asymmetrically affect the degree of similarity between hypotheses and evidence and consequently produce substantial deviations from the predictions of probability theory. In Section II, we observed that the assessment of similarity between hypotheses and evidence affects the perceived support for disjunctive hypotheses, yielding deviations from additivity and monotonicity. In both cases an analysis based on similarity or matching allows insight into a complex pattern of departures from probability theory.

Our studies can be viewed as explorations into the degree to which likelihood judgment obeys various forms of independence. For example, the first section concerns independence based on feature location: are probability ratios invariant as a common feature is moved from evidence to hypotheses? The requirement of additivity of support for disjunctions is another form of independence: does a particular hypothesis evoke the same degree of support when presented by itself as when embedded within a disjunction?

Independence of feature location and additivity of support for disjunctions are two forms of independence required by probability theory but violated in the present data. It may be the case, however, that assessment of support obeys a more fundamental form of independence: context independence across competing hypotheses. Recall that context independence is the requirement that the support for a particular hypothesis does not depend on what other hypotheses are under consideration.

The data presented in Section I bolster the assumption of context independence. We noted that it appears that people do not cancel the common feature residing in two considered hypotheses (e.g., the feature feminist from feminist journalist and feminist realtor). In the presence of cancellation, feminist realtor would be evaluated quite differently in the context of feminist journalist than in the context of Republican journalist, because in the former case the common feature would be cancelled while in the latter it would not. The absence of cancellation thus amounts to a form of context independence. Other work (Brenner \& Rottenstreich, 1998; Fox, 1999) has directly tested context independence and validated it under several conditions.

In short, we observe that support is dependent on feature location and on whether a hypothesis is isolated or combined with others to form a disjunction. However, support for competing hypotheses may be evaluated independently. Context independence is important because it allows pairwise conditional probability judgments to be decomposed into individual assessments of support, which in turn may be related to similarity. The view that support is driven by similarity between hypotheses and evidence provides an organizing principle for understanding the judgment of complex hypotheses and 
how these judgments may be reduced to more primitive constituents of the hypotheses (Asch, 1946; Smith, Osherson, Rips, \& Keane, 1988; Hampton, 1988). Considering support as similarity holds out the prospect of embedding characterizations of judgmental heuristics (such as similarity or representativeness) into a formal structure such as support theory. Models incorporating more precise treatments of similarity (e.g., Tversky, 1977) into support theory provide intriguing avenues for future research.

\section{REFERENCES}

Asch, S. E. (1946). Forming impressions of personality. Journal of Abnormal and Social Psychology, 41, 258-290.

Bar-Hillel, M., \& Fischhoff, B. (1981). When do base rates affect predictions? Journal of Personality \& Social Psychology, 41, 671-680.

Bar-Hillel, M., \& Neter, E. (1993). How alike is it versus how likely is it: A disjunction fallacy in probability judgments. Journal of Personality \& Social Psychology, 65, 1119-1131.

Borgida, E., \& Nisbett, R. E. (1977). The differential impact of abstract vs. concrete information on decisions. Journal of Applied Social Psychology, 7, 258-271.

Brenner, L. A., \& Rottenstreich, Y. (1998). Asymmetric support theory: Focus dependence and context independence in likelihood judgment. Unpublished manuscript.

Chapman, L. J., \& Chapman, J. P. (1967). Genesis of popular but erroneous psychodiagnostic observations. Journal of Abnormal Psychology, 73, 193-204.

Chapman, L. J., \& Chapman, J. P. (1969). Illusory correlation as an obstacle to the use of valid psychodiagnostic signs. Journal of Abnormal Psychology, 74, 271-280.

Fox, C. (1999). Strength of evidence, support, judged probability, and choice under uncertainty. Cognitive Psychology, 38, 167-189.

Gilovich, T., Vallone, R., \& Tversky, A. (1985). The hot hand in basketball: On the misperception of random sequences. Cognitive Psychology, 17, 295-314.

Griffin, D., \& Tversky, A. (1992). The weighing of evidence and the determinants of confidence. Cognitive Psychology, 24, 411-435.

Hampton, J. A. (1988). Disjunction of natural concepts. Memory \& Cognition, 16, 579-591.

Jennings, D., Amabile, T., \& Ross, L. (1982). Informal covariation assessment: Data-based versus theory-based judgments. In D. Kahneman, P. Slovic \& A. Tversky (Eds.), Judgment under uncertainty: Heuristics and biases. Cambridge, England: Cambridge Univ. Press.

Kahneman, D., \& Tversky, A. (1972). Subjective probability: A judgment of representativeness. Cognitive Psychology, 3, 430-454.

Kahneman, D., \& Tversky, A. (1973). On the psychology of prediction. Psychological Review, 80, 237-251.

Kahneman, D., \& Tversky, A. (1982). Variants of uncertainty. Cognition, 11, 143-157.

Kahneman, D., Slovic, P., \& Tversky, A. (1982). Judgment under uncertainty: Heuristics and biases. Cambridge, England: Cambridge Univ. Press.

Rottenstreich, Y., \& Tversky, A. (1997). Unpacking, repacking, and anchoring: Advances in support theory. Psychological Review, 104, 406-415.

Slovic, P., \& Lichtenstein, S. (1968). The relative importance of probabilities and payoffs in risk taking. Journal of Experimental Psychology, 78, 1-18. 
Smith, E. E., Osherson, D. N., Rips, L. J., \& Keane, M. (1988). Combining prototypes: A selective modification model. Cognitive Science, 12, 485-527.

Tversky, A. (1977). Features of similarity. Psychological Review, 84, 327-352.

Tversky, A., \& Kahneman, D. (1971). Belief in the law of small numbers. Psychological Bulletin, 2, 105-110.

Tversky, A., \& Kahneman, D. (1974). Judgment under uncertainty: Heuristics and biases. Science, 185, 1124-1131.

Tversky, A., \& Kahneman, D. (1983). Extensional versus intuitive reasoning: The conjunction fallacy in probability judgment. Psychological Review, 90, 293-315.

Tversky, A., \& Koehler, D. J. (1994). Support theory: A nonextensional representation of subjective probability. Psychological Review, 101, 547-567.

Wagenaar, W., \& Bar-Hillel, M. (1991). Perceptions of randomness. Advances in Applied Mathematics, 12(4), 428-454.

Accepted November 18, 1998 